\title{
ATIVIDADE FÍSICA HABITUAL E CAPACIDADE FUNCIONAL PERCEBIDA DE IDOSAS DO SUL DO BRASIL.
}

\author{
Grasiely Faccin Borges \\ Universidade Federal do Amazonas, Manaus, Amazonas, Brasil
}

Tânia Rosane Bertoldo Benedetti

Universidade Federal de Santa Catarina, Florianópolis, Santa Catarina, Brasil

\section{Sidney Ferreira Farias}

Universidade Federal de Santa Catarina, Florianópolis, Santa Catarina, Brasil

Resumo: O presente estudo teve como objetivo verificar a associação entre o nível de atividade física habitual e a capacidade funcional percebida, de idosas participantes de grupos de convivência. A amostra foi constituída por 92 mulheres com idade entre 60 e 98 anos (Média: 68,1; $\pm 7,0$ anos), participantes de um grupo convivência de idosos de um Município da região Sul do Brasil. Para coleta de dados foi utilizada uma entrevista. Testou-se a associação entre as variáveis através do teste qui- quadrado. Verificou-se associação entre o nível de atividade física habitual e capacidade funcional relatada (QuiQuadrado 18,$9 ; p<0,01)$. A partir deste contexto destaca-se a importância de se manter uma prática regular de atividades físicas, para a manutenção da saúde e de uma boa capacidade funcional.

Palavras-chave: Atividade Motora; Idoso; Centros de Convivência e Lazer

\section{Introdução}

No Brasil, no ano de 2003, a população de 60 anos ou mais era de cerca de 17 milhões de pessoas, representando $10 \%$ da população total do País, e já em 2006, a Pesquisa Nacional por Amostra de Domicílios-PNAD, apontava que os idosos alcançavam, aproximadamente, 19 milhões de pessoas, evidenciando o acelerado processo de envelhecimento da sociedade brasileira (IBGE, 2009).

Apesar da esperança média de vida vir aumentando, advinda dos avanços científicos e médicos para prolongar os anos de vida (CARVALHO, 2006; IBGE, 2009), é importante destacar que no Brasil existe muita desigualdade, aumentando as diferenças entre os idosos com relação entre a funcionalidade física para muitos idosos. Neste contexto, observam-se instituições frágeis, pobreza, desigualdade social e processo de envelhecimento acelerado (BRASIL, 2009).

O conceito de capacidade funcional é bastante complexo abrange reflexões sobre deficiência, incapacidade, desvantagem (JETTE, 1985), e também discussões sobre autonomia e independência (GRIMLEY-EVANS, 1984). A capacidade funcional, em estudos populacionais, tem sido geralmente direcionada em termos da habilidade e independência para realizar determinadas atividades (LIMA-COSTA et al., 2003). A incapacidade funcional no Brasil parece atingir mais as mulheres do que os homens, e também mais os idosos pobres, além disso, está associada com o crescimento da carga de morbidade (BRASIL, 2009).

Pensar a Prática, Goiânia, v. 14, n. 1, p. 1-11, jan./abr. 2011 
Os estudos têm demonstrado que idosas praticantes de atividade física podem melhorar ou manter a capacidade funcional, sendo essa prática um instrumento poderoso para a prevenção e para a promoção da saúde do idoso (MATSUDO et al., 2003).

$\mathrm{O}$ exercício físico e a prática regular de atividade física têm sido apontados como fatores importantes para um envelhecimento saudável (CHODZKO-ZAJKOET al., 2009). Todavia, em recente pesquisa realizada nas capitais brasileiras, o índice de sedentarismo foi de 53,2\% entre as mulheres e 51,5\% entre os homens acima dos 65 anos e, nas atividades físicas de lazer, apenas $20,5 \%$ dos idosos as realizam com frequência suficiente (BRASIL, 2009).

Apesar da Organização Panamericana da Saúde (2005) afirmar que existe uma grande desinformação sobre a saúde do idoso e as particularidades e desafios do envelhecimento populacional, epidemiologicamente já é destacada a importância do nível de atividade física na prevenção e tratamento de incapacidades funcionais em idosos (NELSON et al., 2004).

É importante destacar que no Brasil o número de estudos que abordam o nível de atividade física e a capacidade funcional vem aumentando (FARINASSO et al., 2006; OLIVEIRA et al., 2009), entretanto ainda são necessárias investigações sobre as necessidades reais da população idosa com relação ao nível de atividade física e suas limitações funcionais, principalmente em grupos diferenciados para se destacar quais suas reais necessidades a fim de se obter um melhor resultado em intervenções com esses grupos.

Diante do contexto apresentado, o objetivo desse estudo foi verificar a associação entre o nível de atividade física e a capacidade funcional de idosas participantes de um grupo de convivência.

\section{Métodos}

A composição da amostra foi realizada por meio de conglomerado, em um primeiro momento solicitou-se a lista dos grupos de convivência de idosos cadastrados na secretaria de Ação Social do Município de Maringá/PR. No momento do estudo existiam 4 grupos cadastrados, sendo então realizado um sorteio para seleção de um dos grupos a constituir a amostra desse estudo. Os grupos de convivência ofereciam a oportunidade das idosas participarem, no mínimo, uma vez na semana de atividades como passeio, ginástica, dança, trabalhos manuais entre outras atividades comumente registradas por esses grupos.

Após o sorteio de um grupo, foi solicitada a listagem de nomes das participantes desse grupo de convivência. Dos idosos pertencentes a esse grupo foram excluídos, sujeitos que não aceitassem ou não estivessem em condições para responder a entrevista. $\mathrm{O}$ total de indivíduos entrevistados foi de 92 idosas, com idade entre 60 e 98 anos (Média 68 anos; $\mathrm{DP} \pm 7$ anos).

Instrumentos para Coleta de Dados

Para coleta de dados foi utilizada uma entrevista, composta por três partes. A primeira parte constituída por fatores sóciodemográficos: faixa-etária, estado civil, poder de compra, escolaridade, ocupação e estado de saúde. Para estimar o poder de compra utilizou o critério da ABEP- Associação Brasileira de Empresa de Pesquisas (2003), que estima o poder de compras das pessoas e famílias urbanas, e não tem a pretensão de classificar os indivíduos em classes sociais.

A segunda parte do questionário foi direcionada ao nível de atividade física, onde foi aplicado o International Physical Activity Questionnaire, em sua versão longa- IPAQ/ Versão 8 - adaptada para idosos (BENEDETTI; MAZO; BARRO, 2004), que permite 
estimar o tempo semanal gasto na realização de atividades físicas de intensidade moderada a vigorosa e em diferentes contextos da vida (trabalho, tarefas domésticas, transporte e lazer). Além destas atividades, o IPAQ também possibilita estimar o tempo despendido em atividades passivas (realizadas na posição sentada).

O IPAQ/Versão 8- para idosos divide-se em 13 questões distribuídas por cinco seções:

a) Seção I: Atividade física no trabalho

b) Seção II: Atividade física como meio de transporte

c) Seção III: Atividade física em casa, tarefas domésticas e atenção à família

d) Seção IV: Atividade física de recreação, esporte, exercício e lazer

e) Seção V: Tempo que passa sentada.

Para estimar o nível de atividade física dos idosos utilizou-se o critério de Classificação para Nível de Atividade Física Habitual (NAFH). Para calcular os METs min/sem necessários para a classificação do NAFH, utilizou-se a fórmula sugerida pelo IPAQ Research Committee (2004):

- METs min/sem AF Questão = dias/semana X minutos/dia X MET estimado por intensidade da AF METs min/sem AF por Seção = MET min/sem AF_Q.a + Q.b +Q.c +Q.d - METs min/sem AF Total = MET min/sem AF Ocupacional + Deslocamento + Quintal/Jardim+ Casa + Exercício/Lazer.

O gasto energético estimado em MET, indicado para cada seção de atividade física do IPAQ versão longa, foi sugerido por Craig et al. (2003). Para as atividades de caminhada e andar de bicicleta, utilizou-se o ritmo moderado para o MET estimado. Os MET's estimados foram baseados no Compendium of Physical Activities (Ainsworth et al, 2000).

O tempo que se passa sentado foi analisado separadamente (Craig et al., 2003). Para a classificação do nível de atividade física também foi adotado o consenso obtido entre o Centro de Estudos do Laboratório de Aptidão Física de São Caetano do Sul (CELAFISCS) e o Centers for Disease Control and Prevention (CDC) em 2002. Considerando os critérios de frequência, duração e tipo de atividade física (caminhada, moderada e vigorosa), os indivíduos foram classificados em muito ativos, irregularmente ativos e sedentários (MATSUDO et al., 2002), e também em percetis para a aplicação do qui-quadrado.

Nos resultados apresentados, foram considerados grupos de risco aqueles classificados como sedentários e irregularmente ativos. Segundo a metodologia adotada nesta pesquisa, estes dois grupos, em conjunto, formam o grupo dos insuficientemente ativos.

A terceira parte do questionário tinha como objetivo verificar a capacidade funcional. Utilizou-se a escala de auto-percepção do desempenho em atividades da vida diária, proposta por Andreotti e Okuma (1999).

O instrumento na íntegra foi previamente testado em um estudo piloto e aplicado por um pesquisador previamente instruído e treinado.

Procedimentos para Coleta de Dados

Foram respeitados os princípios éticos e legais, de acordo com as recomendações da Resolução CONEP n. ${ }^{\circ}$ 196/96. Para o início da coleta de dados, foi solicitada autorização da Secretaria de Ação Social do município de Maringá (PR), em seguida buscou-se permissão e apoio dos coordenadores dos grupos de convivência e a aprovação dos protocolos de intervenção do estudo pelo Comitê de Ética em Pesquisa com Seres Humanos, da Universidade Federal de Santa Catarina (parecer consubstanciado- projeto $\left.\mathrm{n}^{\circ} 101 / 05\right)$, todos os participantes assinaram um termo de consentimento livre e esclarecido.

Pensar a Prática, Goiânia, v. 14, n. 1, p. 1-11, jan./abr. 2011 
Análise de Dados

Para a análise dos dados utilizou-se o software EpiData para dar entrada aos dados e o Statística para análise. A descrição dos dados serviu para caracterizar a amostra, com a distribuição em frequência, cálculo de medida de tendência central (média) e de dispersão (desvio-padrão).

Para todos os teste aplicados considerou-se $p<0,05$. Foi aplicado o teste de Kolmogorov-Smirnov nas variáveis do estudo, entretanto os dados não apresentaram um padrão de normalidade, pois $p>0,05$. Sendo assim para verificar associações entre as variáveis utilizou-se a estatística não- paramétrica, aplicando análise de significância de associação, para isso utilizou-se também o teste qui-quadrado.

Para verificar a associação entre o nível de atividade física e capacidade funcional os dados foram organizados de acordo com os percentis 30, 50 e 70 de cada variável, com o fim de homogeneizar os pontos de cortes. A partir destas categorias, testou-se a associação entre as variáveis através do teste qui-quadrado (Tabela 03).

\section{Resultados}

Das idosas participantes de grupo de convivência, $87 \%$ não completaram o ensino fundamental. A principal fonte de renda é a aposentadoria $(39,1 \%)$. A maioria era dona de casa $(40,2 \%)$ e pertencente à classe $C(46,7 \%)$ com uma renda média familiar de 927 reais, seguidas por $28,3 \%$ na classe D (até 207 reais mensais), e $25 \%$ das idosas foram classificadas entre as classes B1, B2 e A2 que tem como renda média familiar de 1.669 a 4.648 reais mensais (tabela 01) ABEP (2003).

Tabela 01. Distribuição das características sócio-demográficas de uma amostra de idosas $(\mathrm{n}=92)$ participantes de grupo de convivência na cidade de Maringá/PR.

\begin{tabular}{lll}
\hline Característica & Frequência & Percentual (100\%) \\
\hline Escolaridade & & \\
Analfabeta ou fundamental incompleto & 80 & 87,0 \\
Fundamental completo & 6 & 6,5 \\
Médio completo & 5 & 5,4 \\
Superior completo & 1 & 1,1 \\
Ocupação & & \\
Aposentada, mas trabalha & 7 & 7,6 \\
Só aposentada & 36 & 39,1 \\
Só dona de casa & 37 & 40,2 \\
Pensionista & 12 & 13,0 \\
Classe Econômica (ABEP) & & \\
A2 & 3 & 3,3 \\
B1 & 5 & 5,4 \\
B2 & 15 & 16,3 \\
C & 43 & 46,7 \\
D & 34 & 28,3 \\
\hline
\end{tabular}

Quanto aos aspectos referentes a atividade física habitual, observou-se que, de uma forma geral, as idosas entrevistadas podem ser consideradas em $83,7 \%$ como ativas ou muito ativas, $14,1 \%$ como irregularmente ativas e $2,2 \%$ como sedentárias.

Sobre o comportamento das idosas em relação às atividades físicas habituais, no

Pensar a Prática, Goiânia, v. 14, n. 1, p. 1-11, jan./abr. 2011 
somatório das atividades realizadas no trabalho, transporte, atividades domésticas e de lazer encontrou-se um gasto médio energético de 2.211,8 MET/min $/ \mathrm{sem}$. $(\mathrm{DP} \pm 1346,9 \mathrm{MET} / \mathrm{min} / \mathrm{sem})$, esse valor não contempla o tempo gasto sentado.

Com relação ao tempo gasto em cada atividade em uma semana, verificou-se que a atividade que as idosas gastaram menos tempo foi a atividade de trabalho (Média 50,16 min; $\mathrm{DP} \pm 180,54 \mathrm{~min}$ ) o gasto energético dessa atividade pelas idosas teve uma média de 220,2 $\mathrm{MET} / \mathrm{min} / \mathrm{sem}, \mathrm{DP} \pm 885,2 \mathrm{MET} / \mathrm{min} / \mathrm{sem}$, já o tempo gasto sentadas, apesar de ser avaliado separadamente, foi o maior segundo relatado pelas idosas (Média 1150,97 min; $\mathrm{DP} \pm 552,6 \mathrm{~min}$ ), onde tiveram um gasto energético médio de $1703 \mathrm{MET} / \mathrm{min} / \mathrm{sem}$ $\mathrm{DP} \pm 766,9 \mathrm{MET} / \mathrm{min} / \mathrm{sem})$.

Com relação à capacidade funcional das idosas, verificou-se que apresentam um percentual de $81,5 \%$ com uma capacidade funcional muito boa, enquanto outras $14,1 \%$ com uma capacidade boa e apenas 4,3\% com uma capacidade média, nenhuma das entrevistadas encontrava-se na classificação ruim.

Tabela 02. Frequência de respostas de atividades da vida diária que as idosas $(n=92)$, participantes de grupos de convivência, relataram maior dificuldade em realizar (Escala de auto-percepção do desempenho em atividades da vida diária proposta por Andreotti e Okuma (1999).

\begin{tabular}{lcc}
\hline Atividades & Frequência n=92 & $\mathbf{\% ~ ( 1 0 0 \% ) ~}$ \\
\hline Subir uma escadaria (mais de 40 degraus) & 66 & 71,73 \\
Descer uma escadaria (mais de 40 degraus) & 58 & 63,04 \\
Levantar do Chão & 54 & 58,69 \\
Andar em subidas & 51 & 55,43 \\
Andar 10-12 quarteirões & 47 & 51,00 \\
Cortar a unha do pé & 46 & 50,00 \\
Deitar no Chão & 46 & 50,00 \\
Andar depressa & 46 & 50,00 \\
Subir uma escada de 15-20 degraus & 44 & 47,82 \\
Descer uma escada de 15-20 degraus & 42 & 45,65 \\
Segurar objetos pesados & 30 & 32,60 \\
\hline
\end{tabular}

Entre as atividades nas quais as idosas apresentaram maiores dificuldades para realizar estão subir uma escada (mais de 40 degraus) com $71,73 \%$ dos relatos. Numa proporção decrescente, a segunda atividade mais citada foi descer escadas com mais de 40 degraus $(63,04 \%)$ e a terceira foi levantar-se do chão $(58,69 \%)$. A maioria destas atividades exige um nível mais elevado de esforço físico e a utilização de grandes grupos musculares e principalmente dos membros inferiores (tabela 02 ).

Com relação a associação entre o nível de atividade física e a capacidade funcional das idosas percebeu-se por meio de teste de qui-quadrado uma associação significativa $(p<0,001$, Qui-Quadrado=18,978) (tabela 03).

Pensar a Prática, Goiânia, v. 14, n. 1, p. 1-11, jan./abr. 2011 
Tabela 03. Distribuição entre classes segundo o nível de atividade física habitual e a capacidade funcional percebida de acordo, de acordo, com os percentis 30, 50 e 70 das 92 idosas participantes de grupo de convivência do município de Maringá/PR.

\begin{tabular}{c|c|c|c}
\hline & \multicolumn{3}{|c}{ Capacidade Funcional* } \\
\hline & $\begin{array}{c}\text { Até 138,9 } \\
31,5 \%(29)\end{array}$ & $\begin{array}{c}\text { De 138,9 a 148,50 } \\
40,2 \%(37)\end{array}$ & $\begin{array}{c}\text { De 148,50 a 155 } \\
28,3 \%(26)\end{array}$ \\
\hline Atividade Física** & & & \\
\hline Até 1614 & $63,0 \%(17)$ & $25,9 \%(7)$ & $9,1 \%(3)$ \\
$29,3 \%(27)$ & $15,4 \%(6)$ & $51,3 \%(20)$ & $33,3 \%(13)$ \\
\hline $\begin{array}{c}\text { De 1614 até 2022 } \\
\text { 42,4\%(39) }\end{array}$ & & $38,5 \%(10)$ & $38,5 \%(10)$ \\
\hline $\begin{array}{c}\text { De 2022 até 2851,20 } \\
\text { 28,3\%(26) }\end{array}$ & $23,1 \%(6)$ & & \\
\hline
\end{tabular}

$p<\mathbf{0 , 0 0 1}$ Qui-quadrado $=\mathbf{1 8 , 9 7 8}$

*Capacidade Funcional medida em pontos de acordo com o questionário de Andreotti e Okuma (1999).

**Atividade Física medida em MET/min/sem

\section{Discussão}

A diversidade de instrumentos e formas utilizadas para medir ou avaliar a capacidade funcional e níveis de atividade física em idosos dificulta a comparação dos resultados.

Com relação à tabela 03 , quando verificou-se a associação entre a prática de atividade física e a capacidade funcional, os resultados encontrados corroboram com os resultados citados anteriormente pela literatura (BRACH ET AL., 2003; STESSMAN et al., 2002; SULANDER et al., 2005).

Os grupos de convivência parecem estimular a prática regular de atividades físicas aliadas a outras atividades, influenciando positivamente nos níveis de atividade física habitual e também na capacidade funcional das idosas.

Algumas características sócio-econômicas das idosas entrevistadas foram semelhantes ao de um estudo com mulheres do município de São Paulo (ROSA et al., 2003), como apresentado em nossos resultados a maioria eram donas de casa e aposentadas, e estas características foram entendidas no estudo citado acima, como indicadores de risco de dependência moderada ou grave, apesar das características convergirem a um risco, essa dependência não ocorreu em nosso estudo (tabela 01 ). $\mathrm{O}$ aspecto que diferenciou nossos resultados do estudo de Rosa et al., (2003), foi o fato das idosas do Grupo de Convivência praticarem exercícios físicos dentro do próprio grupo, além disso estavam envolvidas em atividades sociais, evidenciando a importância desse fator na manutenção da autonomia e consequentemente na capacidade de realizar as atividades da vida diária.

Os resultados referentes à capacidade funcional entre as diferentes regiões e estados brasileiros, encontram maior prevalência de incapacidade funcional entre mulheres idosas na região Norte $(19,6 \%)$ e menor na região Sul (14,7\%) (PARAHYBA; VERAS; MELZER, 2005). Outro fator importante de se destacar é que em pesquisa domiciliar a prevalência de capacidade funcional inadequada é mais elevada (FIELDER; PERES, 2008). Diferente de estudos de base domiciliar, nosso estudo apontou que idosas participantes do grupo de convivência apresentam uma capacidade funcional elevada.

Pensar a Prática, Goiânia, v. 14, n. 1, p. 1-11, jan./abr. 2011 
Os resultados encontrados com relação à capacidade funcional das idosas, aproximam-se dos resultados apresentados por Matsudo et al. (2003). Nesse estudo as idosas eram praticantes de um programa de exercícios físico e também relataram realizar a maioria das atividades da vida diária sem nenhum tipo de ajuda ou grau de dificuldade.

Os grupos de convivência não só estimulam atividades físicas como envolvem os idosos em outras atividades sociais e intelectuais. Em uma pesquisa que acompanhou por 8 anos, 814 idosos, em uma zona urbana do Japão, observou-se que o baixo nível de envolvimento social, e atividade intelectual pode preceder a uma incapacidade funcional (FUJIWARA et al., 2002).

Quando verificamos as atividades que as idosas relataram ter maior dificuldade em realizar, verificamos que subir escadas também foi uma das atividades relatadas no estudo de Parahyba, Veras e Melzer (2005) com idosas brasileiras, principalmente quando comparou a estudos realizados na Inglaterra onde essa dificuldade não aparecia com tanta frequência. Diante desses dados podemos levantar a hipótese de que o idoso brasileiro parecem apresentar um padrão diferente de atividades físicas diárias quando comparados com os relatos dos idosos estudados na Inglaterra, fato esse que destaca a importância de se dar valor para as atividades físicas que venham melhorar a capacidade funcional do idoso levando em conta suas reais necessidades.

Outro aspecto interessante sobre as atividades da vida diária, é a dificuldade relatada para caminhar de 10 a 12 quarteirões, que corrobora com os resultados apresentados por Lima-Costa et al. (2003), a partir dos dados do PNAD de 1998, onde os idosos também relataram dificuldade nessa tarefa. Essa habilidade é de extrema importância e pode ser usada como determinante em relação o status funcional (FERRUCCI et al., 2000). Em um estudo que acompanhou 226 idosas, verificou-se que as pessoas que apresentavam uma fadiga contínua ou dificuldade em realizar atividades, entre 75 a 80 anos, tiveram um aumento significativo do risco de declínio funcional e da mortalidade entre os 80 e 85 anos de idade (AVLUND; PEDERSEN; SCHROLL, 2003). Levando em consideração as dificuldades relatadas, pessoas com alguma dificuldade em realizar atividades da vida diária, tem maiores riscos de agravamento desse problema nas idades mais avançadas, sendo de grande importância uma maior atenção prévia, onde as medidas podem ser mais simples e efetivas.

Apesar de a idade ser um fator que se relaciona com a perda da capacidade funcional, o envelhecimento não é e não pode ser entendido como sinônimo de incapacidade funcional, visto que podemos encontrar mulheres acima de 85 anos reportando não ter dificuldades em realizar as atividades da vida diária (FIELDER; PERES, 2008; PARAHYBA; VERA; MELZER, 2005). Em nosso estudo, 81\% das idosas foram classificadas com capacidade funcional muito boa e nenhuma foi classificada com a capacidade funcional ruim.

Com relação ao nível de atividade física, é importante destacar que qualquer tipo de atividade física é melhor do que levar uma vida sedentária. Assim como sugerido nos resultados encontrados sobre a associação do nível de atividade física habitual e a capacidade funcional nas idosas participantes de grupos de convivência (tabela 03), a prática regular da atividade física vem sendo considerada como protetora das limitações, apresentando também um melhor beneficio em relação à capacidade física nas idades mais avançadas (BRACH et al; 2003).

Outro fator que é interessante considerar é que o envolvimento com atividade física pode estar atrelado ao grupo de convivência, esse tipo de atividade aparece com uma frequência de uma a três vezes por semana, e pode estar relacionado com o efeito na manutenção da capacidade funcional da amostra estudada, esse tipo de comportamento e

Pensar a Prática, Goiânia, v. 14, n. 1, p. 1-11, jan./abr. 2011 
resultados também foram evidenciados por Matsudo et al. (2003).

\section{Conclusão}

Considerando os resultados do estudo podemos considerar que pode existir uma associação entre o nível de atividade física e a capacidade funcional. As idosas participantes do grupo de convivência possuem níveis elevados de atividade física e capacidade funcional.

A partir deste contexto destaca-se a grande importância de se manter uma prática regular de exercícios físicos, que como resultados apresentados nesse estudo, mesmo com o surgimento de características que as colocariam em grupo de risco para a incapacidade funcional isso não ocorreu. Sendo assim, os níveis de atividade física habitual das idosas parecem ser importantes para a manutenção de uma boa capacidade funcional.

\section{Agradecimentos}

Agradecemos ao Professor Dr. Jair Sindra Virtuoso Júnior, por suas importantes contribuições para a realização deste trabalho.

\section{Referências}

AINSWORTH, B. E.; HASKELL, W. L.; WHITT, M. C.; IRWIN, M. L.; SWARTZ, A. M.; STRATH, S. J.; O'BRIEN, W. L.; BASSETT, D. R. Jr.; SCHMITZ, K. H.; EMPLAINCOURT, P. O.; JACOBS, D. R. Jr.; LEON, A. S. Compendium of Physical Activities: An update of activity codes and MET intensities. Medicine and Science in Sports and Exercise, v.32, Suppl.p.S498-S516, 2000.

ANDREOTTI, R. A.; OKUMA, S.S. Validação de uma bateria de testes de atividades da vida diária para idosos fisicamente independentes. Revista Paulista Educação Física, São Paulo, v.13, n. 1, p. 46- 66, 1999.

AVLUND, K.; PEDERSEN, A. N.; SCHROLL, M. Functional Decline From Age 80 to 85: Influence of Preceding Changes in Tiredness in Daily Activities. Psychosomatic Medicine, v. 65, p.771-777, 2003.

BENEDETTI, T. B.; MAZO, G. Z.; BARROS, M.V.G. Aplicação do questionário internacional de atividades físicas para avaliação do nível de atividades físicas de mulheres idosas: validade concorrente e reprodutibilidade teste-reteste. Revista Brasileira Ciência e Movimento, v. 12, n.1, p. 25-34, 2004.

BRACH, J. S.; GERALD, S. F.; NEWMAN, A. B.; KELSEY, S.; KULLER, L.; VANSWEARINGEN, J. M.; KRISKA, A. M. Physical Activity and Functional Status in Community- Dwelling Older Women. A 14-Year Prospective Study. Archives of Internal Medicine, v. 163, p.2565-2571, 2003.

BRASIL. Ministério da Saúde. Secretaria de Vigilância em Saúde. Secretaria de Gestão Estratégica e Participativa. Vigitel Brasil 2008: vigilância de fatores de risco e proteção para doenças crônicas por inquérito telefônico / Ministério da Saúde, Secretaria de Vigilância em Saúde, Secretaria de Gestão Estratégica e Participativa, Brasília: Ministério

Pensar a Prática, Goiânia, v. 14, n. 1, p. 1-11, jan./abr. 2011 
da Saúde, 2009.

CARVALHO, M. J. A Actividade Física na Terceira Idade e relações intergeracionais. Revista Brasileira de Educação Física e Esporte, v. 20, n.5 suplemento, p.71-72, setembro, 2006.

CHODZKO-ZAJKO, W. J.; FIATARONE SINGH, M. A.;MINSON, C. T.; NIGG, C. R.; SALEM G. J.; SKINNER, J. S. American College of Sports Medicine Position Stand: Exercise and Physical Activity for Older Adults, p.1510-1530, 2009.

CRAIG, C. L.; MARSHALL, A. L.; SJOSTROM, M.; BAUMAN, A. E.; BOOTH, M. L.; AINSWORTH, B. E. et al. International physical activity questionnaire: 12-country reliability and validity. Medicine Science Sports and Exercise, v. 35, n.8, p.1381-95, 2003.

FARINASSO, A. L. C.; MARQUES, S.; RODRIGUES, R. A. P.; HAAS, V. J. Capacidade funcional e morbidades referidas de idosos em uma área de abrangência do PSF. Revisa Gaúcha de Enfermagem, Porto Alegre (RS), v.27, n.1, p.45-52, março, 2006.

FERRUCCI, L.; BANDINELLI, S.; BENVENUTI, E.; IORIO, A.; MACCHI, C.; HARRIS, T. B.; GURALNIK, J. M. Subsystems contributing to the decline in ability to walk: bridging the gap between epidemiology and geriatric practice in the In CHIANTI study. Journal of the American Geriatrics Society, n. 48, p.1618-1625, 2000.

FIELDER, M. M; PERES, K. G. Capacidade funcional e fatores associados em idosos do Sul do Brasil: um estudo de base populacional. Cadernos de Saúde Pública, n. 24, v. 2, p.409-415, 2008.

FUJIWARA ,Y. ; SHINKAI, S.; KUMAGA ,S.; AMANO, H.; YOSHIDA, Y.; YOSHIDA, H.; KIM, H.; KIM, T.; ISHIZAKI, T.; HAGA, H.; WATANABE, S.; SHIBATA, H. Longitudinal changes in higher-level functional capacity of an older population living in a Japanese urban community. Archives of Gerontology and Geriatrics, v. 36, p. 141-153, 2003.

GRIMLEY-EVANS, J. Prevention of age-associated loss of autonomy: epidemiological approaches. Journal of Chronic Disease, v. 37, p. 353-363, 1984.

JETTE, A. M., BRANCH, L. Impairment and disability in the aged. Journal Chronic Disease, v. 38, p.59-65, 1985.

IBGE. Estudos \& Pesquisas: Informação Demográfica e Sócioeconômica- Indicadores Sociodemográficos e de Saúde no Brasil, n.25, 2009.

LIMA- COSTA, M. F.; BARRETO, S. M.; GIATTI, L. Condições de saúde, capacidade funcional, uso de serviços de saúde e gastos com medicamentos da população idosa brasileira: um estudo descritivo baseado na Pesquisa Nacional por Amostra de Domicílios. Caderno de Saúde Pública, Rio de Janeiro, v. 19, n.3, p.735-743, 2003. 
10.5216/rpp.v14i1.12314

MATSUDO, S. M.; MATSUDO, V. R.; BARROS NETO, T. L.; ARAUJO, T. L. Evolução do perfil neuromotor e capacidade funcional de mulheres fisicamente ativas de acordo com a idade cronológica. Revista Brasileira de Medicina do Esporte, Niterói, v. 9, n. 6, Nov/dez, 2003.

MATSUDO, S. M.; MATSUDO, V. R.; ARAÚJO, T.; ANDRADE, D.; ANDRADE, E.; OLIVEIRA, L. et al. Nível de atividade física da população do Estado de São Paulo: análise de acordo com o gênero, idade e nível sócio-econômico, distribuição geográfica e de conhecimento. Revista Brasileira de Ciências e Movimento, v. 10, p. 41-50, 2002.

NELSON ME, REJESKI J, BLAIR SN, DUCAN PW, JUDGE JO, KING AC, MACERA CA, CASTANEDA- SCEPPA C. Physical Activity and Public Health in Older Adults: recommendation from the American College of Sports Medicine and the American Heart Association. Circulation, v. 116, p. 1094-1105, 2007.

OLIVEIRA, D. V.; BENEDETI, M. R.; MORALES, R. C.; FARIA, T. G. Análise da Capacidade Funcional de Idosos Institucionalizados á partir da auto-avaliação. Conexões: revista da Faculdade de Educação Física da UNICAMP, Campinas, v. 7, n. 2, p. 79-95, maio/ago. 2009.

ORGANIZAÇÃO PAN- AMERICANA DA SAÚDE - World Health Organization. Envelhecimento ativo: uma política de saúde, p. 16, 2005.

PARAHYBA, M. I.; VERAS, R.; MELZER, D. Incapacidade funcional entre as mulheres idosas no Brasil. Revista de Saúde Pública, São Paulo, v. 39, n.3, p. 383-390, jun., 2005.

ROSA, C. E.; BENÍCIO, M. H. D; LATORRE, M. R. D. O.; RAMOS, L. R. Fatores determinantes da capacidade funcional entre idosos. Revista de Saúde Pública, v.37, n.1, p.40-48, 2003.

STESSMAN, J.; HAMMERMAN-ROZENBERG, R.; MAARAVI, Y.; COHEN, A. Effect of Exercise on Ease in Performing Activities of Daily Living and Instrumental Activities of Daily Living From Age 70 to 77: The Jerusalem Longitudinal Study. American Geriatrics Society, v. 50, p.1934-1938, 2002.

SULANDER, T.; MARTELIN, T.; RAHKONEN, O; NISSINEN, A.; UUTELA, A. Associations of functional ability with health-related behavior and body mass index among the elderly. Archives of Gerontology and Geriatrics, v 40, p.185-199, 2005.

\section{PHYSICAL ACTIVITY AND FUNCTIONAL DISABILITY REPORTED OF IN OLDER IN SOUTHERN BRAZIL.}

Abstract: The aim of this study was to verify the association between level of physical activity and perceived functional capacity of senior citizens members of groups of elderly. The sample consisted of 92 women aged between 60 and 98 years (mean: $68.1 \pm 7.0$ years) participating in a group of elderly living in a city located in southern Brazil. To collect data we used an interview form. We tested the association between variables using the chisquare. Was founded an association between the level of habitual physical activity and

Pensar a Prática, Goiânia, v. 14, n. 1, p. 1-11, jan./abr. 2011 
10.5216/rpp.v14i1.12314

functional ability reported (Chi-square $18.9 ; p<0.01$ ). From this context we highlight the importance of maintaining a regular practice of physical activities for maintaining good health and functional capacity.

Keyword: Motor Activity; Aged; Centers of Connivance and Leisure

\section{LA ACTIVIDAD FÍSICA HABITUAL Y LA PERCEPCIÓN DE COMPETENCIA FUNCIONAL DE LOS ANCIANOS DEL SUR DE BRASIL.}

Resumen: El objetivo de este estudio fue verificar la asociación de nivel de actividad física entre la percepción y la capacidad funcional de los miembros más antiguos de los grupos comunitarios participantes. La muestra estuvo constituida por 92 mujeres de edades comprendidas entre 60 y 98 años (media: 68,1 \pm 7,0 años) Participar en un grupo de ancianos que viven en una ciudad situada en el sur de Brasil. Para recoger los datos se utilizó una entrevista. Pusimos a prueba la asociación entre las variables mediante el chicuadrado. Una asociación entre el nivel de actividad física habitual y capacidad comunicadas funcional (Chi-cuadrado $=18,9 ; p<0,001$ ). Desde este contexto, destacamos la importancia de mantener una práctica regular de actividades físicas para mantener la buena salud y capacidad funcional.

Palabras clave: Actividad Motora; Anciano; Centros de Ocio y Convivencia

Endereço para correspondência:

Grasiely Faccin Borges

grasiely.borges@gmail.com

Universidade Federal do Amazonas

Instituto de Saúde e Biotecnologia- ISB

Estrada Coari/Mamiá

69460-000 - Coari, AM - Brasil

Pensar a Prática, Goiânia, v. 14, n. 1, p. 1-11, jan./abr. 2011 